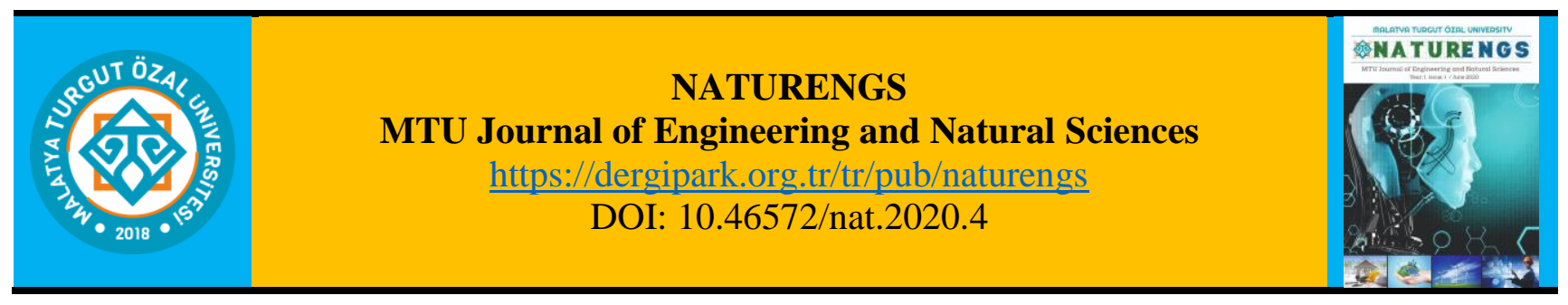

\title{
New Analytical Solutions for Space and Time Fractional Phi-4 Equation
}

\author{
Ayșegül DAŞCIOĞLU ${ }^{1}$, Sevil ÇULHA ÜNAL ${ }^{2 *}$, Dilek VAROL BAYRAM ${ }^{3}$ \\ 1,2,3 Department of Mathematics, Faculty of Science and Arts, Pamukkale University, Denizli, Turkey.
}

(Received: 03.03.2020; Accepted: 18.05.2020)

\begin{abstract}
In this paper, the largest set in the literature of space, time and space-time conformable fractional Phi-4 equations is found by utilizing an analytical method based upon the Jacobi elliptic functions. These solutions are obtained in a general form including trigonometric, rational, complex and hyperbolic functions. Some problems are presented to illustrate the practical application of the proposed method and some of the solutions are also demonstrated with the two-dimensional and three-dimensional graphics.
\end{abstract}

Mathematics Subject Classification: 35G20, 35L05, 35R11, 33E05.

Keywords: Jacobi elliptic functions; Space-time fractional Phi-4 equation; Conformable fractional derivative; Analytic method.

\section{INTRODUCTION}

The Phi-4 equation which is a particular form of Klein-Gordon equation and has used in particle and nuclear physics is defined by

$$
u_{t t}-a u_{x x}-b u+\lambda u^{3}=0
$$

where $a, b$ and $\lambda$ are constants. This equation has also been the subject of intensive investigation for classical and quantized field theory, and it has kink-like solutions which are not solitons [1]. Besides, the Phi- 4 equation has been mostly examined as the easiest nontrivial relativistic invariant field theoretical model [2]. If $\lambda=0$, the above equation turns into a linear differential equation.

Recently, various methods have been used to solve the integer order Phi-4 equation. The solution methods for this equation are sine-cosine [3], sine-cosine ansantz [4], tanh [4], generalized tanh [5], modified extended tanh-function [6], Weierstrass elliptic function [7], Jacobi-Gauss-Lobatto collocation [8], variational [9], modified simple equation [10,11], homotopy perturbation [12], homotopy analysis [12], Adomian decomposition [12], $\left(G^{\prime} / G, 1 / G\right)$-expansion [13], trigonometric B-Spline collocation [14] generalized Kudyashov [15] and improved $F$-expansion [16].

In recent years, searching solutions of the fractional differential equations have drawn remarkable interest not only for applied mathematicians but also for the other scientists. So far, various solutions of the time fractional Phi-four equation have been founded by utilizing the tanh function [17], modified residual power series [18], modified Kudryashov [19], exponential 
function [19], extended direct algebraic [20], mapping [21], modified mapping [21], qhomotopy analysis transform ( $q$-HATM) [22], and generalized Kudryashov [23] methods. The solutions of space-time conformable fractional Phi-four equation is also gained by using the $\left(G^{\prime} / G, 1 / G\right)$-expansion [24] method. Among these methods, exponential function, the modified Kudryashov, extended direct algebraic, mapping, modified mapping and generalized Kudryashov methods contain the fractional derivatives with respect to time in the conformable sense, while the $\left(G^{\prime} / G, 1 / G\right)$-expansion method includes the fractional derivatives with respect to space and time in the conformable sense. However, a solution method for space fractional Phi-4 equations is not yet available in the literature. On the other side, although there has not found any method involving the Jacobi elliptic functions for the solutions of the conformable space-time fractional Phi-4 equation, Jacobi elliptic functions have been used to obtain the exact solutions of different conformable fractional partial differential equations [2534].

In this study, Jacobi elliptic functions have been used to improve an analytic method for the space, time, and space-time conformable fractional Phi-4 equations is presented. Our goal is to find the possible largest set of exact solutions of the space-time conformable fractional Phi-four equation in the form

$D_{t}^{\alpha} D_{t}^{\alpha} u-a D_{x}^{\beta} D_{x}^{\beta} u-b u+\lambda u^{3}=0, \quad 0<\alpha, \beta \leq 1$

Here, $D_{x}^{\beta}$ and $D_{t}^{\alpha}$ stand for the conformable fractional derivative of the unknown function $u(x, t)$ with respect to $x$ and $t$, respectively.

The rest of the paper has been organized as follows: In the second section, conformable fractional derivative and some properties of Jacobi elliptic functions are presented. The exact solutions of the conformable space-time fractional Phi-four equation are obtained in terms of Jacobi elliptic functions in the third section. In the fourth section, to illustrate the practical application of the proposed method, four problems are presented and some solutions are demonstrated by the 2D and 3D graphics. Finally, the paper has been concluded in the fifth section.

\section{PRELIMINARIES}

Recently, Khalil et al. [35] has described the conformable fractional derivative. Since it is similar to the definition of the usual derivative this definition of the fractional derivative is the simplest way compared to the other fractional derivatives. Thus, the fractional Phi-4 equation is examined in the conformable sense in this paper. The definition and some of the properties of the mentioned conformable fractional derivative can be given as follows and other properties of it can be seen in [35] and [36].

Definition [35]: Let $f:[0, \infty) \rightarrow \mathbb{R}$ be a function. The conformable fractional derivative of $\alpha$ the order of the function $f$ is described by

$$
T_{\alpha}(f)(t)=\lim _{\varepsilon \rightarrow 0} \frac{f\left(t+\varepsilon t^{1-\alpha}\right)-f(t)}{\varepsilon}, \quad \alpha \in(0,1), \quad t>0 .
$$

If the function $f$ is $\alpha$-differentiable in conformable sense and $\lim _{t \rightarrow 0^{+}} f^{(\alpha)}(t)$ exists, then $f^{(\alpha)}(0)=\lim _{t \rightarrow 0^{+}} f^{(\alpha)}(t)$. 
Theorem 1 [35]: Let the functions $f$ and $g$ be $\alpha$-differentiable in conformable sense for $t>0$ such that $\alpha \in(0,1]$. Then, the following properties are satisfied:

1) $T_{\alpha}(a f+b g)=a T_{\alpha}(f)+b T_{\alpha}(g) ; \quad \forall a, b \in \mathbb{R}$.

2) $T_{\alpha}\left(t^{p}\right)=p t^{p-\alpha} ; \quad \forall p \in \mathbb{R}$.

3) $T_{\alpha}(\lambda)=0$, where $\lambda$ is a constant.

4) $T_{\alpha}(f g)=f T_{\alpha}(g)+g T_{\alpha}(f)$.

5) $T_{\alpha}\left(\frac{f}{g}\right)=\frac{g T_{\alpha}(f)-f T_{\alpha}(g)}{g^{2}}$.

6) If $f$ is differentiable, then $T_{\alpha}(f)(t)=t^{1-\alpha} \frac{d f}{d t}(t)$.

Theorem 2 [36]: Assume that the functions $f, g:(0, \infty) \rightarrow \mathbb{R}$ be $\alpha$-differentiable in conformable sense and $0<\alpha \leq 1$. Suppose that $h(t)=f(g(t))$, then the composite function $h(t)$ is $\alpha$-differentiable in conformable sense also. For non-zero $t$ and $g(t) \neq 0$, we obtain

$$
T_{\alpha}(h)(t)=T_{\alpha}(f)(g(t)) \cdot T_{\alpha}(g)(t) \cdot g(t)^{\alpha-1} .
$$

If $t=0$, we get

$$
T_{\alpha}(h)(0)=\lim _{t \rightarrow 0} T_{\alpha}(f)(g(t)) \cdot T_{\alpha}(g)(t) \cdot g(t)^{\alpha-1} .
$$

The basic Jacobi elliptic functions are

$$
\operatorname{sn} \xi=\operatorname{sn}\left(\xi \mid m^{2}\right), \operatorname{cn} \xi=\operatorname{cn}\left(\xi \mid m^{2}\right), \operatorname{dn} \xi=\operatorname{dn}\left(\xi \mid m^{2}\right)
$$

where $m$ is a complex number and it represents the modulus of the elliptic function. If this modulus is a real number, it can always be set up as $0<m^{2}<1$. Besides these three wellknown elliptic functions, there are nine other elliptic functions named as ns, nc, nd, sc, sd, cd, cs, ds and dc which are determined by taking reciprocals and quotients [37]. Twelve Jacobi elliptic functions are examined in four groups, and the notations can be clear from Table 1.

Table 1. Jacobi elliptic functions

\begin{tabular}{|c|c|c|c|}
\hline $\mathbf{1}$ & $\mathbf{s n} \xi$ & $\mathbf{c n} \xi$ & $\mathbf{d n} \xi$ \\
\hline $\mathbf{2}$ & $\operatorname{sd} \xi=\frac{\operatorname{sn} \xi}{\mathrm{dn} \xi}$ & $\operatorname{cd} \xi=\frac{\mathrm{cn} \xi}{\mathrm{dn} \xi}$ & $\mathrm{nd} \xi=\frac{1}{\mathrm{dn} \xi}$ \\
\hline $\mathbf{3}$ & $\operatorname{sc} \xi=\frac{\operatorname{sn} \xi}{\operatorname{cn} \xi}$ & $\mathrm{nc} \xi=\frac{1}{\operatorname{cn} \xi}$ & $\mathrm{dc} \xi=\frac{\mathrm{dn} \xi}{\operatorname{cn} \xi}$ \\
\hline $\mathbf{4}$ & $\mathrm{ns} \xi=\frac{1}{\operatorname{sn} \xi}$ & $\operatorname{cs} \xi=\frac{\operatorname{cn} \xi}{\operatorname{sn} \xi}$ & $\mathrm{ds} \xi=\frac{\mathrm{dn} \xi}{\operatorname{sn} \xi}$ \\
\hline
\end{tabular}

These double periodic functions satisfy the properties in Table 2. Moreover, when $m=0$ and $m=1$, Jacobi elliptic functions transform trigonometric and hyperbolic functions, respectively. The derivatives and the other properties of these functions can be seen in Ref. [38]. 
Table 2. The relations of Jacobi elliptic functions

\begin{tabular}{|l|l|l|l|l|}
\hline $\mathbf{1}$ & $\mathbf{s n}^{2} \xi+\mathbf{c n}^{2} \xi=\mathbf{1}$ & $\mathbf{d n}^{2} \xi+\boldsymbol{m}^{2} \mathbf{s n}^{2} \xi=\mathbf{1}$ & $\mathbf{d n}^{2} \xi-\boldsymbol{m}^{2} \mathbf{c n}^{2} \xi=\mathbf{1}-\boldsymbol{m}^{2}$ & $\mathbf{c n}^{2} \xi+\left(\mathbf{1}-\boldsymbol{m}^{2}\right) \mathbf{s n}^{2} \xi=\mathbf{d n}^{2} \xi$ \\
\hline $\mathbf{2}$ & $\mathrm{nd}^{2} \xi-m^{2} \mathrm{sd}^{2} \xi=1$ & $\mathrm{~cd}^{2} \xi+\left(1-m^{2}\right) \mathrm{sd}^{2} \xi=1$ & $m^{2} \mathrm{~cd}^{2} \xi+\left(1-m^{2}\right) \mathrm{nd}^{2} \xi=1$ & $\mathrm{~cd}^{2} \xi+\mathrm{sd}^{2} \xi=\mathrm{nd}^{2} \xi$ \\
\hline $\mathbf{3}$ & $\mathrm{nc}^{2} \xi-\mathrm{sc}^{2} \xi=1$ & $\mathrm{dc}^{2} \xi-\left(1-m^{2}\right) \mathrm{sc}^{2} \xi=1$ & $\mathrm{dc}^{2} \xi-\left(1-m^{2}\right) \mathrm{nc}^{2} \xi=m^{2}$ & $\mathrm{nc}^{2} \xi-m^{2} \mathrm{sc}^{2} \xi=\mathrm{dc}^{2} \xi$ \\
\hline $\mathbf{4}$ & $\mathrm{ns}^{2} \xi-\mathrm{cs}^{2} \xi=1$ & $\mathrm{~ns}^{2} \xi-\mathrm{ds}^{2} \xi=m^{2}$ & $\mathrm{ds}^{2} \xi-\mathrm{cs}^{2} \xi=1-m^{2}$ & $m^{2} \mathrm{cs}^{2} \xi+\left(1-m^{2}\right) \mathrm{ns}^{2} \xi=\mathrm{ds}^{2} \xi$ \\
\hline
\end{tabular}

\section{EXACT SOLUTIONS OF THE SPACE-TIME FRACTIONAL PHI-4 EQUATION}

In this part of the paper, the space and time conformable fractional Phi-four equation (1) is considered. Utilizing the following change of the variables

$$
\xi=k \frac{t^{\alpha}}{\alpha}+l \frac{x^{\beta}}{\beta}
$$

such that $k$ and $l$ are arbitrary constants and using the chain rule, Eq. (1) becomes an ordinary differential equation in the form

$$
\left(k^{2}-a l^{2}\right) \frac{d^{2} u}{d \xi^{2}}-b u+\lambda u^{3}=0
$$

where $k^{2}-a l^{2} \neq 0$. When $\lambda=0$, Eq. (2) becomes a linear ordinary differential equation, and the solution can be founded easily. In this study, the solutions are investigated for the nonlinear case.

The main purpose of this analytical method is to gain the solutions $u(\xi)$ in the form

$$
u(\xi)=\sum_{j=0}^{N} c_{j} F^{j}(\xi) .
$$

Here, $c_{j}$ are the coefficients to be determined and $F(\xi)$ are the solutions of the auxiliary nonlinear ordinary differential equation given by

$$
(d F / d \xi)^{2}=P F^{4}(\xi)+Q F^{2}(\xi)+R
$$

where $P, Q$ and $R$ are constants. The solutions of this auxiliary equation are in terms of Jacobi elliptic functions. Depending on the selected values of the constants $P, Q$ and $R$, the set of these Jacobi elliptic function solutions of Eq. (3) are given in Table 3. Some of them can be seen also in Ref. [31-34]. 
Table 3. The solution $F$ for $P, Q$ and $R$

\begin{tabular}{|c|c|c|c|c|}
\hline & $\boldsymbol{P}$ & $Q$ & $\boldsymbol{R}$ & $\boldsymbol{F}$ \\
\hline 1 & $m^{2}$ & $-\left(m^{2}+1\right)$ & 1 & $\pm \operatorname{sn} \xi, \pm \operatorname{cd} \xi$ \\
\hline 2 & 1 & $-\left(m^{2}+1\right)$ & $m^{2}$ & $\pm n s \xi, \pm d c \xi$ \\
\hline 3 & $-m^{2}$ & $-\left(m^{2}+1\right)$ & -1 & $\pm i \operatorname{sn} \xi, \quad \pm i \operatorname{cd} \xi$ \\
\hline 4 & -1 & $-\left(1+m^{2}\right)$ & $-m^{2}$ & $\pm i n s \xi, \pm i \mathrm{dc} \xi$ \\
\hline 5 & 1 & $-m^{2}+2$ & $1-m^{2}$ & $\pm \operatorname{cs} \xi, \pm i \operatorname{dn} \xi$ \\
\hline 6 & $-m^{2}+1$ & $-m^{2}+2$ & 1 & $\pm \mathrm{sc} \xi, \pm i n d \xi$ \\
\hline 7 & -1 & $2-m^{2}$ & $m^{2}-1$ & $\pm i c s \xi, \quad \pm \operatorname{dn} \xi$ \\
\hline 8 & $-1+m^{2}$ & $-m^{2}+2$ & -1 & $\pm i s c \xi, \quad \pm \operatorname{nd} \xi$ \\
\hline 9 & $1-m^{2}$ & $2 m^{2}-1$ & $-m^{2}$ & $\pm \mathrm{nc} \xi, \pm i m s d \xi$ \\
\hline 10 & $-m^{2}$ & $2 m^{2}-1$ & $1-m^{2}$ & $\pm \operatorname{cn} \xi, \pm \frac{i}{m} \mathrm{ds} \xi$ \\
\hline 11 & $m^{2}-1$ & $2 m^{2}-1$ & $m^{2}$ & $\pm \operatorname{inc} \xi, \pm m s d \xi$ \\
\hline 12 & $m^{2}$ & $2 m^{2}-1$ & $m^{2}-1$ & $\pm i \operatorname{cn} \xi, \quad \pm \frac{1}{m} \mathrm{ds} \xi$ \\
\hline 13 & $m^{4}-m^{2}$ & $2 m^{2}-1$ & 1 & $\pm \frac{i}{m} \mathrm{nc} \xi, \quad \pm \mathrm{sd} \xi$ \\
\hline 14 & 1 & $2 m^{2}-1$ & $m^{4}-m^{2}$ & $\pm i m \mathrm{cn} \xi, \pm \mathrm{ds} \xi$ \\
\hline 15 & $-m^{4}+m^{2}$ & $2 m^{2}-1$ & -1 & $\pm \frac{1}{m} \mathrm{nc} \xi, \quad \pm i \mathrm{sd} \xi$ \\
\hline 16 & -1 & $2 m^{2}-1$ & $-m^{4}+m^{2}$ & $\pm m \operatorname{cn} \xi, \quad \pm i \mathrm{ds} \xi$ \\
\hline 17 & $\frac{1}{4}$ & $\frac{1+m^{2}}{2}$ & $\frac{\left(1-m^{2}\right)^{2}}{4}$ & 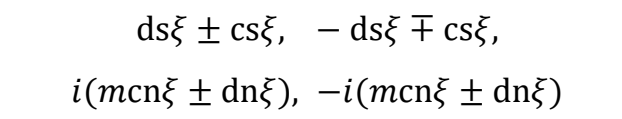 \\
\hline 18 & $\frac{\left(1-m^{2}\right)^{2}}{4}$ & $\frac{1+m^{2}}{2}$ & $\frac{1}{4}$ & 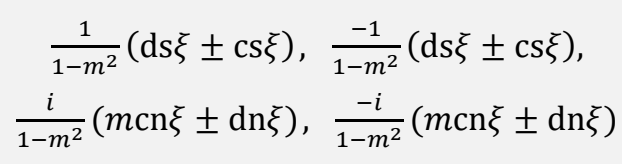 \\
\hline 19 & $-\frac{1}{4}$ & $\frac{1+m^{2}}{2}$ & $-\frac{\left(1-m^{2}\right)^{2}}{4}$ & $\begin{array}{l}i(\mathrm{ds} \xi \pm \operatorname{cs} \xi),-i(\mathrm{ds} \xi \pm \operatorname{cs} \xi) \\
m \mathrm{cn} \xi \pm \operatorname{dn} \xi,-m \operatorname{cn} \xi \mp \mathrm{dn} \xi\end{array}$ \\
\hline 20 & $-\frac{\left(1-m^{2}\right)^{2}}{4}$ & $\frac{1+m^{2}}{2}$ & $-\frac{1}{4}$ & 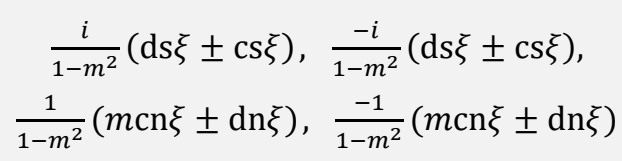 \\
\hline 21 & $\frac{-m^{2}+1}{4}$ & $\frac{m^{2}+1}{2}$ & $\frac{-m^{2}+1}{4}$ & $\begin{array}{c}\mathrm{nc} \xi \pm \mathrm{sc} \xi,-\mathrm{nc} \xi \mp \mathrm{sc} \xi \\
i(m \mathrm{sd} \xi \pm \mathrm{nd} \xi),-i(m \mathrm{sd} \xi \pm \mathrm{nd} \xi)\end{array}$ \\
\hline 22 & $\frac{-1+m^{2}}{4}$ & $\frac{m^{2}+1}{2}$ & $\frac{-1+m^{2}}{4}$ & $\begin{array}{l}i(\mathrm{nc} \xi \pm \mathrm{sc} \xi),-i(\mathrm{nc} \xi \pm \mathrm{sc} \xi) \\
m s d \xi \pm \mathrm{nd} \xi,-m \mathrm{sd} \xi \mp \mathrm{nd} \xi\end{array}$ \\
\hline 23 & $\frac{1}{4}$ & $\frac{m^{2}-2}{2}$ & $\frac{m^{4}}{4}$ & $\begin{array}{c}\mathrm{ns} \xi \pm \mathrm{d} s \xi,-\mathrm{ns} \xi \mp \mathrm{ds} \xi \\
\mathrm{dc} \xi \pm \sqrt{1-m^{2}} \mathrm{nc} \xi,-\mathrm{dc} \xi \mp \sqrt{1-m^{2}} \mathrm{nc} \xi\end{array}$ \\
\hline
\end{tabular}




\begin{tabular}{|c|c|c|c|c|}
\hline 24 & $\frac{m^{4}}{4}$ & $\frac{m^{2}-2}{2}$ & $\frac{1}{4}$ & $\begin{array}{cl}\frac{1}{m^{2}}(\mathrm{~ns} \xi \pm \mathrm{ds} \xi), & \frac{-1}{m^{2}}(\mathrm{~ns} \xi \pm \mathrm{ds} \xi) \\
\frac{1}{m^{2}}\left(\mathrm{dc} \xi \pm \sqrt{1-m^{2}} \mathrm{nc} \xi\right), & \frac{-1}{m^{2}}\left(\mathrm{dc} \xi \pm \sqrt{1-m^{2}} \mathrm{nc} \xi\right)\end{array}$ \\
\hline 25 & $-\frac{1}{4}$ & $\frac{m^{2}-2}{2}$ & $-\frac{m^{4}}{4}$ & $\begin{array}{c}i(\mathrm{~ns} \xi \pm \mathrm{ds} \xi),-i(\operatorname{ns} \xi \pm \mathrm{ds} \xi) \\
i\left(\mathrm{dc} \xi \pm \sqrt{1-m^{2}} \mathrm{nc} \xi\right),-i\left(\operatorname{dc} \xi \pm \sqrt{1-m^{2}} \mathrm{nc} \xi\right)\end{array}$ \\
\hline 26 & $-\frac{m^{4}}{4}$ & $\frac{m^{2}-2}{2}$ & $-\frac{1}{4}$ & 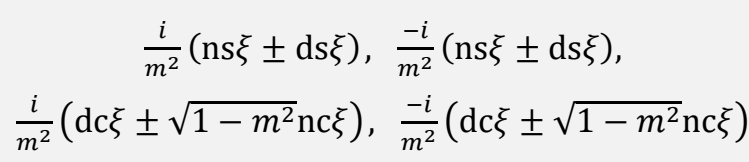 \\
\hline 27 & $\frac{m^{2}}{4}$ & $\frac{m^{2}-2}{2}$ & $\frac{m^{2}}{4}$ & $\begin{array}{c}\operatorname{sn} \xi \pm i \operatorname{cn} \xi,-\operatorname{sn} \xi \mp i \operatorname{cn} \xi \\
\operatorname{cd} \xi \pm i \sqrt{1-m^{2}} \operatorname{sd} \xi,-\operatorname{cd} \xi \mp i \sqrt{1-m^{2}} \operatorname{sd} \xi\end{array}$ \\
\hline 28 & $-\frac{m^{2}}{4}$ & $\frac{m^{2}-2}{2}$ & $-\frac{m^{2}}{4}$ & $\begin{array}{c}\operatorname{cn} \xi \pm i \operatorname{sn} \xi,-\operatorname{cn} \xi \mp i \operatorname{sn} \xi \\
\sqrt{1-m^{2}} \operatorname{sd} \xi \pm i \operatorname{cd} \xi,-\sqrt{1-m^{2}} \operatorname{sd} \xi \mp i \operatorname{cd} \xi\end{array}$ \\
\hline 29 & $\frac{1}{4}$ & $\frac{1-2 m^{2}}{2}$ & $\frac{1}{4}$ & $\begin{array}{c}\mathrm{ns} \xi \pm \operatorname{cs} \xi,-\operatorname{ns} \xi \mp \operatorname{cs} \xi \\
m \operatorname{sn} \xi \pm i \mathrm{dn} \xi,-m \operatorname{sn} \xi \mp i \operatorname{dn} \xi \\
\mathrm{dc} \xi \pm \sqrt{1-m^{2}} \mathrm{sc} \xi,-\operatorname{dc} \xi \mp \sqrt{1-m^{2}} \operatorname{sc} \xi \\
m \operatorname{cd} \xi \pm i \sqrt{1-m^{2}} \mathrm{nd} \xi,-m \operatorname{cd} \xi \mp i \sqrt{1-m^{2}} \mathrm{nd} \xi\end{array}$ \\
\hline 30 & $-\frac{1}{4}$ & $\frac{1-2 m^{2}}{2}$ & $-\frac{1}{4}$ & 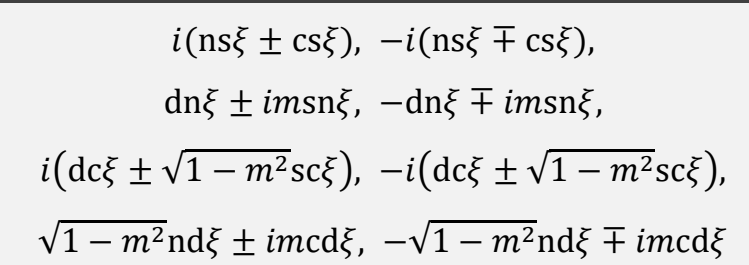 \\
\hline
\end{tabular}

By applying a balancing procedure to the highest orders of nonlinear and linear terms, we obtain the number $N=1$. Hence, the solution of Eq. (2) can be stated as

$$
u(\xi)=c_{0}+c_{1} F
$$

Since the ordinary differential equation (2) is a second order differential equation, we differentiate this solution two times and using the derives form of Eq. (3), we have

$$
u^{\prime \prime}(\xi)=c_{1} Q F+2 c_{1} P F^{3} .
$$

Substituting Eq. (4) into Eq. (2), a third order polynomial in $F$ is gained. After that, setting its coefficients to be zero, the following equations system arises

$$
\begin{aligned}
-b c_{0}+\lambda c_{0}^{3} & =0 \\
k^{2} Q c_{1}-a l^{2} Q c_{1}+3 \lambda c_{0}^{2} c_{1}-b c_{1} & =0 \\
3 \lambda c_{0} c_{1}^{2} & =0 \\
2 k^{2} P c_{1}-2 a l^{2} P c_{1}+\lambda c_{1}^{3} & =0 .
\end{aligned}
$$


Solving this system, we get $c_{0}=0, c_{1}=\mp \sqrt{-2 b P /(\lambda Q)}$ and $c_{0}=\mp \sqrt{b / \lambda}, c_{1}=0$ such that

$$
Q=b /\left(k^{2}-a l^{2}\right)
$$

If we take $A=\sqrt{b / \lambda}$, the solutions of Eq. (2) become

$$
u= \pm A \sqrt{\frac{-2 P}{Q}} F, \quad u= \pm A
$$

Substituting the function $F$ from Table 3 into the above solution and taking inverse transformation, the solutions of Eq. (1) are obtained. Moreover, the elementary function solutions of Eq. (2) can be found by utilizing the Jacobi elliptic functions for $m=0$ and $m=$ 1. Some of these solutions are constant such that $u=0$ and $u= \pm A$, the other solutions are listed in Table 4.

Table 4. Nonconstant solutions of Eq. (2) when $m=0$ and $m=1$

\begin{tabular}{|l|l|}
\hline $\boldsymbol{m}=\mathbf{0}$ & $\boldsymbol{m}=\mathbf{1}$ \\
\hline$u= \pm \sqrt{2} A \sec \xi$ & $u= \pm \sqrt{2} A \operatorname{sech} \xi$ \\
$u= \pm \sqrt{2} A \csc \xi$ & $u= \pm i \sqrt{2} A \operatorname{csch} \xi$ \\
$u= \pm i A \tan \xi$ & $u= \pm A \tanh \xi$ \\
$u= \pm i A \cot \xi$ & $u= \pm A \operatorname{coth} \xi$ \\
$u=i A(\sec \xi \pm \tan \xi)$ & $u=A(i \operatorname{sech} \xi \pm \tanh \xi)$ \\
$u=-i A(\sec \xi \pm \tan \xi)$ & $u=-A(i \operatorname{sech} \xi \pm \tanh \xi)$ \\
$u=i A\left(\frac{\sin \xi}{1 \pm \cos \xi}\right)=i A(\csc \xi \mp \cot \xi)$ & $u=A\left(\frac{\sinh \xi}{\cosh \xi \pm 1}\right)=A(\operatorname{coth} \xi \mp \operatorname{csch} \xi)$ \\
$u=-i A\left(\frac{\sin \xi}{1 \pm \cos \xi}\right)=-i A(\csc \xi \mp \cot \xi)$ & $u=-A\left(\frac{\sinh \xi}{\cosh \xi \pm 1}\right)=-A(\operatorname{coth} \xi \pm \operatorname{csch} \xi)$ \\
\hline
\end{tabular}

\section{APPLICATIONS}

In that part of the paper, four different types of examples are considered. These examples are composed of time, space and space-time fractional types of Phi-4 Eq. (1). The solutions of the given examples are also demonstrated by graphics. In all figures, the solutions are drawn by the Mathematica 11.3.

Example 1. Consider the conformable space-time fractional Phi-4 Eq. (1) for $a=1, b=1$, $\lambda=1$ and $\alpha=0.5, \beta=1$; that is

$$
D_{t}^{1 / 2} D_{t}^{1 / 2} u-u_{x x}-u+u^{3}=0 .
$$

This equation is called the time fractional Phi-4 equation. The solutions of Eq. (6) are

$$
u= \pm \sqrt{\frac{-2 P}{Q}} F, \quad u= \pm 1
$$


such that $A=1$. When $m=1$, condition (5) is satisfied for $k=1 / 2$ and $l=\sqrt{3} / 2$, then transformation becomes $\xi=\sqrt{t}+(\sqrt{3} / 2) x$. In Table 4 , when $m=1$, the solutions in case 1 and 3 become $u= \pm \tanh (\sqrt{t}+(\sqrt{3} / 2) x)$. These solutions are illustrated for $-20 \leq x \leq 20$ and $0 \leq t \leq 20$ in Figure 1 and Figure 2. The $( \pm)$ signs correspond to localized soliton solutions that move with opposite screw senses. They have also named a kink soliton and an antikink soliton, respectively [39]. Thus, Figure 1 represents the kink type travelling wave solution, while Figure 2 represents the antikink type travelling wave solution. Besides, Figure 3 and Figure 4 illustrate the same solutions with a two-dimensional plot for $-20 \leq x \leq 20$ at time $t=2$.

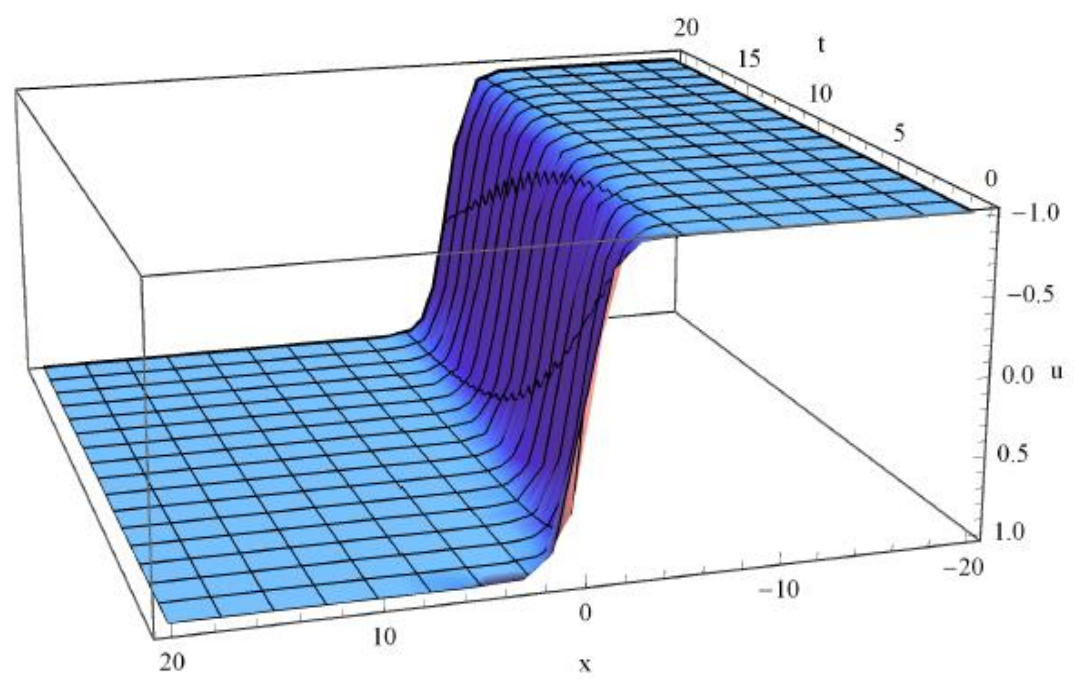

Figure 1. 3D plot of solution $u(x, t)=\tanh (\sqrt{t}+(\sqrt{3} / 2) x)$

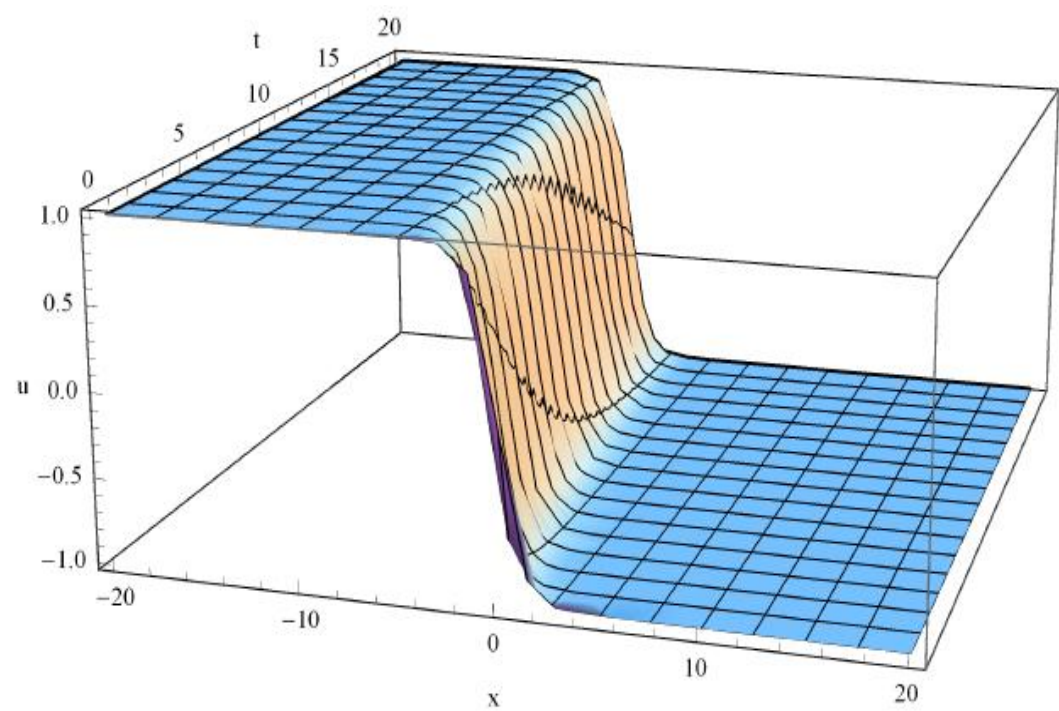

Figure 2. 3D plot of solution $u(x, t)=-\tanh (\sqrt{t}+(\sqrt{3} / 2) x)$ 


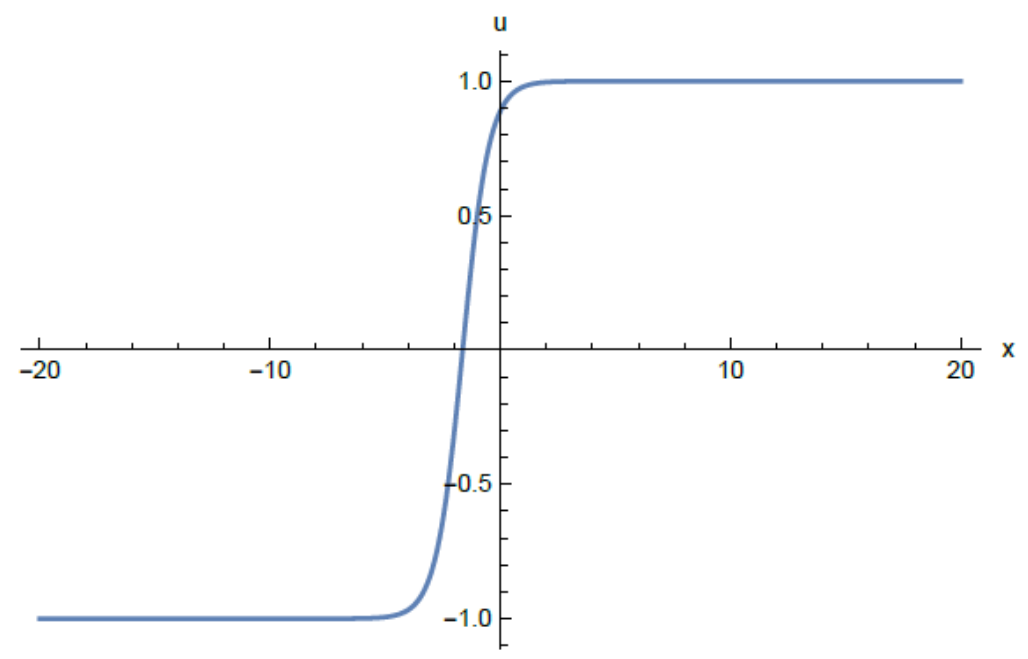

Figure 3. 2D plot of $u(x, t)=\tanh (\sqrt{2}+(\sqrt{3} / 2) x)$

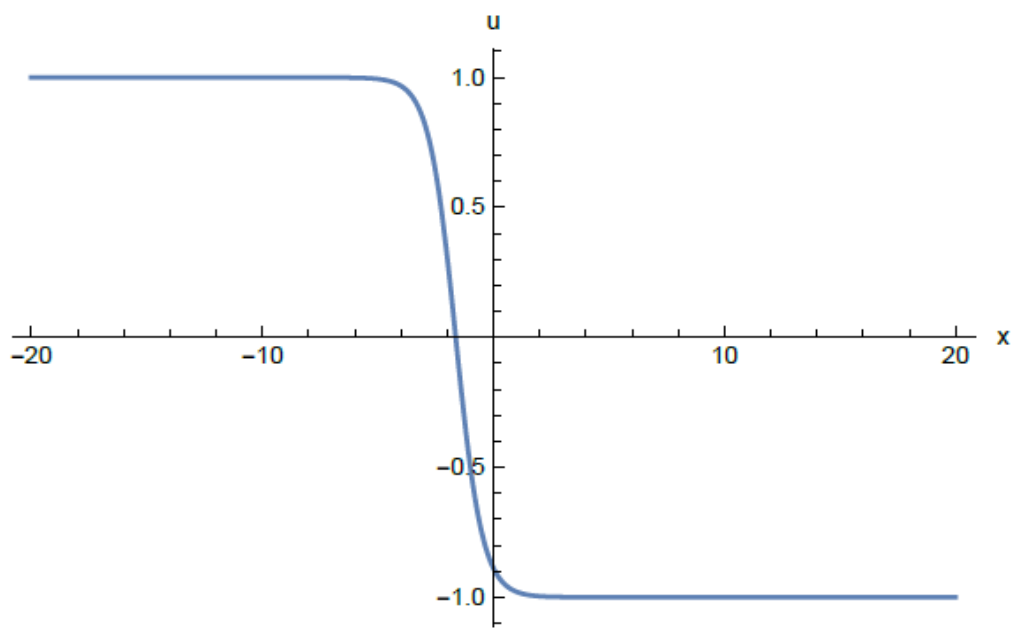

Figure 4. 2D plot of $u(x, t)=-\tanh (\sqrt{2}+(\sqrt{3} / 2) x)$

Example 2. Consider the conformable space-time fractional Phi-4 Eq. (1) for $a=5, b=-2$, $\lambda=2$ and $\alpha=0.5, \beta=1$; that is

$$
D_{t}^{1 / 2} D_{t}^{1 / 2} u-5 u_{x x}+2 u+2 u^{3}=0
$$

This equation is called the time fractional Phi-4 equation. The solutions of Eq. (7) are

$$
u= \pm i \sqrt{\frac{-2 P}{Q}} F, \quad u= \pm i
$$

such that $A=i$. When $m=0$, condition (5) is satisfied for $k=l=1$, then transformation becomes $\xi=x+2 \sqrt{t}$. In Table 4, when $m=0$, the solutions in case 17 and 19 become $u=$ $\mp(\csc (x+2 \sqrt{t}) \pm \cot (x+2 \sqrt{t}))$. Here, there are four different solutions, but we only consider two of them. Figure 5 and Figure 6 demonstrate the following solutions

$$
u=\mp(\csc (x+2 \sqrt{t})+\cot (x+2 \sqrt{t}))
$$


in the region $-10 \leq x \leq 10$ and $1 \leq t \leq 2$. Figure 5 represents the kink type travelling wave solution, while Figure 6 represents the antikink type travelling wave solution. Besides, Figure 7 and Figure 8 illustrate the same solutions with a 2D plot for $-10 \leq x \leq 10$ at $t=2$. Furthermore, the graphics of the solutions $u=-\csc (x+2 \sqrt{t})+\cot (x+2 \sqrt{t})$ and $u=$ $\csc (x+2 \sqrt{t})-\cot (x+2 \sqrt{t})$ are similar to $u=\csc (x+2 \sqrt{t})+\cot (x+2 \sqrt{t})$ and $u=$ $-\csc (x+2 \sqrt{t})-\cot (x+2 \sqrt{t})$, the only difference between the graphics is that 3.5 units are shifted to the right. Therefore,

$$
u=\mp \csc (x+2 \sqrt{t})-\cot (x+2 \sqrt{t})
$$

are called antikink type solutions while

$$
u=\mp \csc (x+2 \sqrt{t})+\cot (x+2 \sqrt{t})
$$

are called kink type solutions.

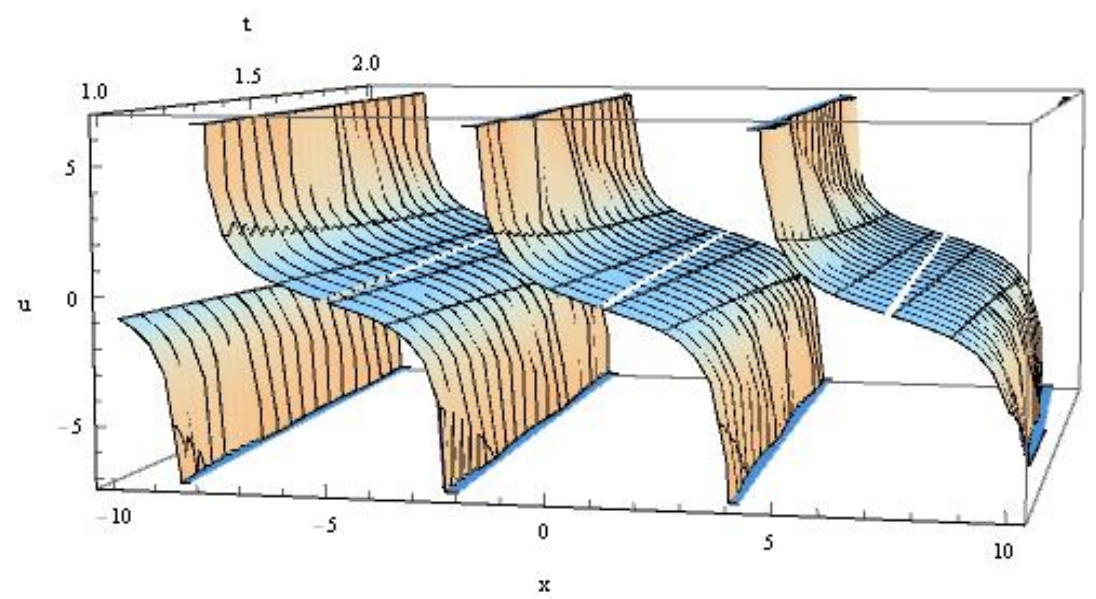

Figure 5. 3D plot of solution $u(x, t)=\csc (x+2 \sqrt{t})+\cot (x+2 \sqrt{t})$

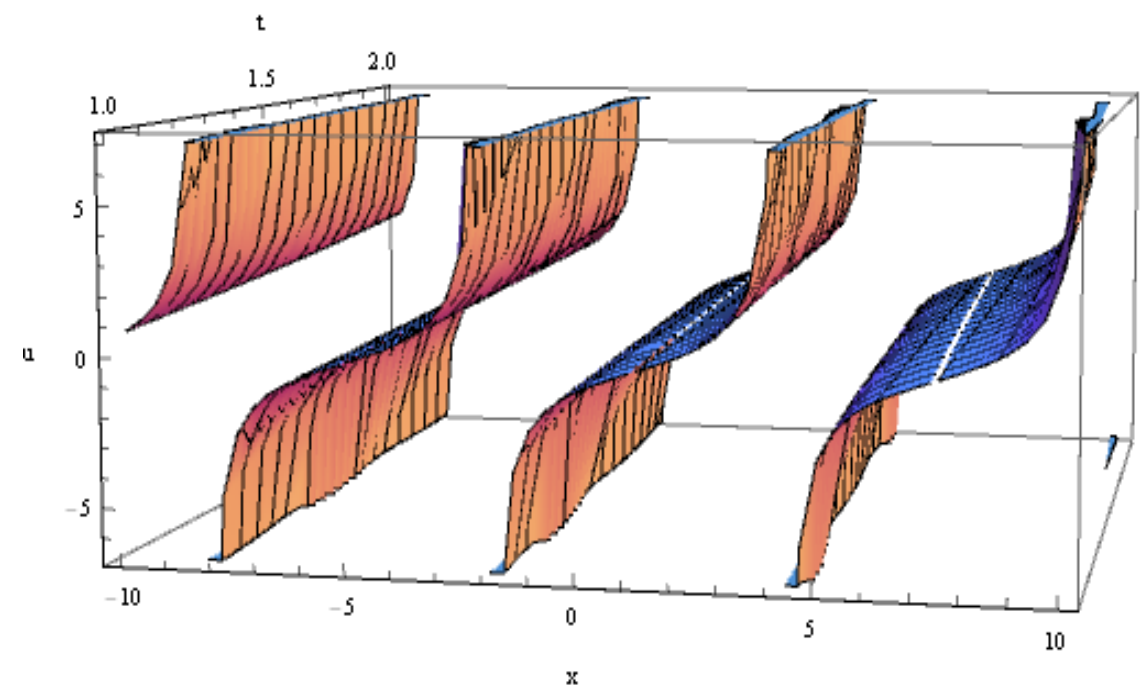

Figure 6. 3D plot of solution $u(x, t)=-\csc (x+2 \sqrt{t})-\cot (x+2 \sqrt{t})$ 


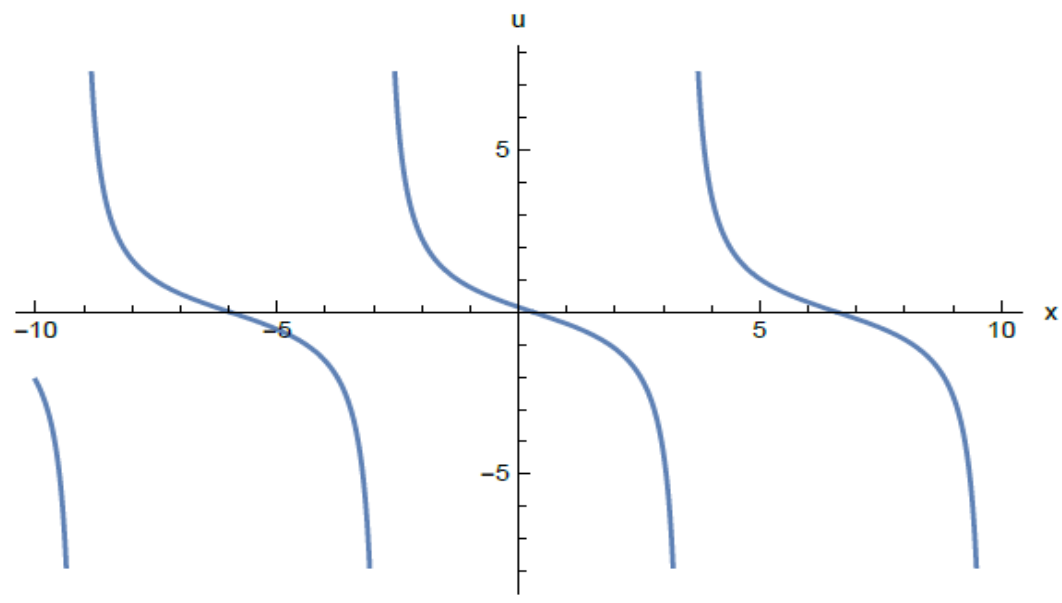

Figure 7. 2D plot of $u(x, t)=\csc (x+2 \sqrt{2})+\cot (x+2 \sqrt{2})$

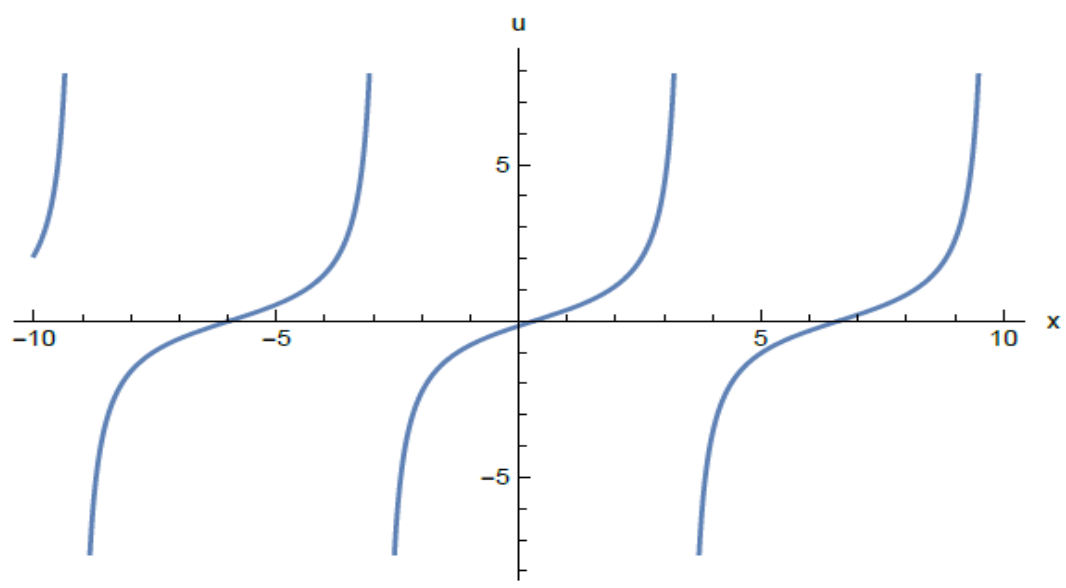

Figure 8. 2D plot of $u(x, t)=-\csc (x+2 \sqrt{2})-\cot (x+2 \sqrt{2})$

Example 3. Consider the conformable space-time fractional Phi-4 Eq. (1) for $a=-3, b=4$, $\lambda=2$ and $\alpha=1, \beta=0.5$; that is

$$
u_{t t}+3 D_{x}^{1 / 2} D_{x}^{1 / 2} u-4 u+2 u^{3}=0 .
$$

This equation is called the space fractional Phi-4 equation. The solutions of Eq. (8) are

$$
u= \pm \sqrt{\frac{-4 P}{Q} F}, \quad u= \pm \sqrt{2}
$$

such that $A=\sqrt{2}$. When $m=1$, condition (5) is satisfied for $k=l=1$, then transformation becomes $\xi=t+2 \sqrt{x}$. In Table 4, when $m=1$, the solutions in case 10, 12, 14 and 16 become $u= \pm 2 \operatorname{sech}(t+2 \sqrt{x})$. These solutions are illustrated for $5 \leq x \leq 10$ and $0 \leq t \leq 20$ in Figure 9 and Figure 10. Furthermore, Figure 11 and Figure 12 demonstrate the same solutions with the two-dimensional plot for $0 \leq x \leq 20$ at $t=3$. 


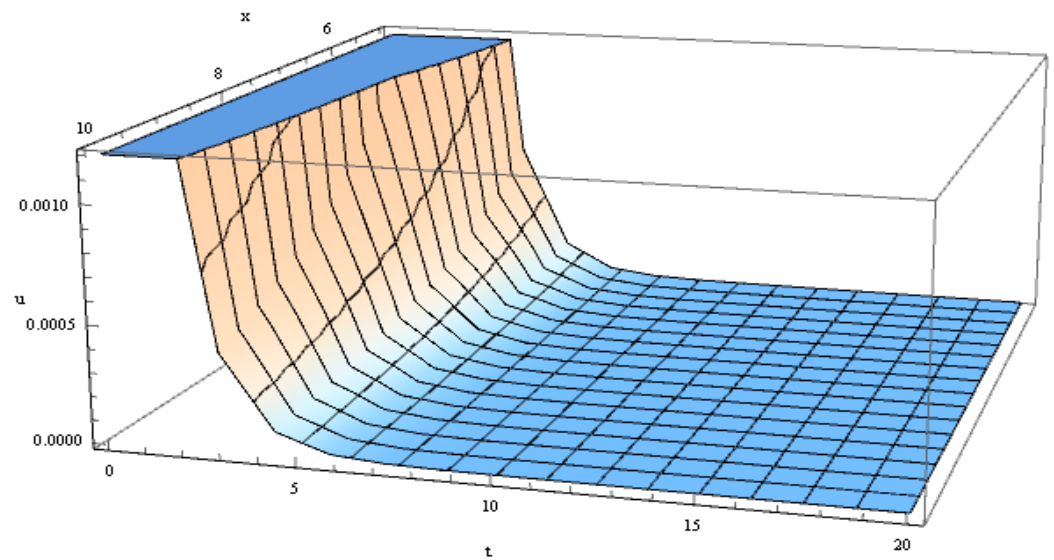

Figure 9. 3D plot of solution $u(x, t)=2 \operatorname{sech}(t+2 \sqrt{x})$

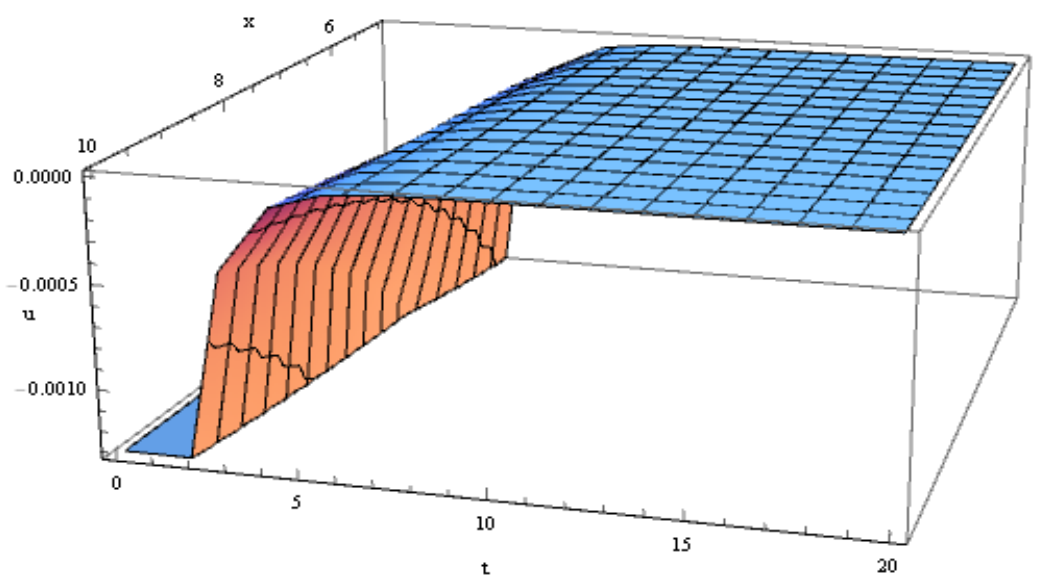

Figure 10. 3D plot of solution $u(x, t)=-2 \operatorname{sech}(t+2 \sqrt{x})$

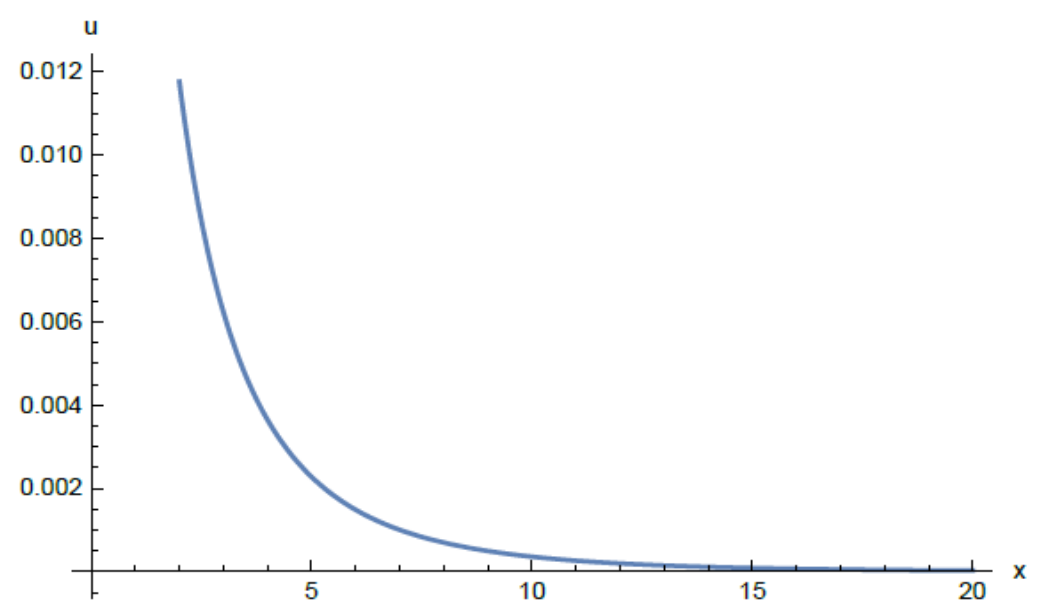

Figure 11. 2D plot of $u(x, t)=2 \operatorname{sech}(3+2 \sqrt{x})$ 


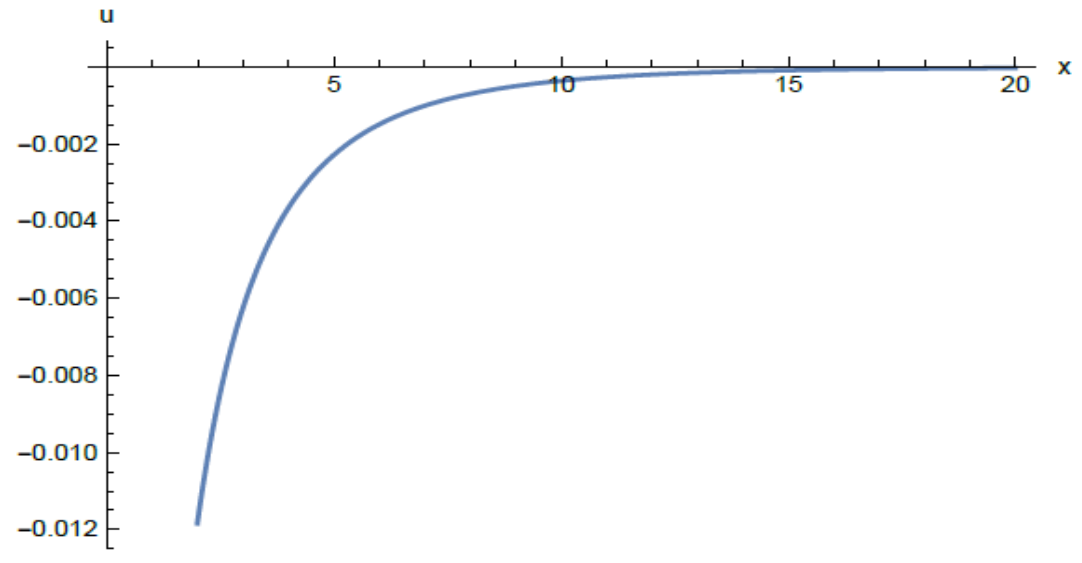

Figure 12. $2 \mathrm{D}$ plot of $u(x, t)=-2 \operatorname{sech}(3+2 \sqrt{x})$

Example 4. Consider the conformable space-time fractional Phi-4 Eq. (1) for $a=1, b=16$, $\lambda=-16$ and $\alpha=0.2, \beta=0.2$; that is

$$
D_{t}^{1 / 5} D_{t}^{1 / 5} u-D_{x}^{1 / 5} D_{x}^{1 / 5} u-16 u-16 u^{3}=0 .
$$

This equation is called the space-time fractional Phi-4 equation. The solutions of Eq. (9) are

$$
u= \pm i \sqrt{\frac{-2 P}{Q}} F, \quad u= \pm i
$$

such that $A=i$. When $m=0$, condition (5) is satisfied for $k=3$ and $l=1$, then transformation becomes $\xi=15 \sqrt[5]{t}+5 \sqrt[5]{x}$ In Table 4, when $m=0$, the solutions in case 6 and 8 become $u=\mp \tan (15 \sqrt[5]{t}+5 \sqrt[5]{x})$. These solutions are illustrated for $0 \leq x \leq 20$ and $0 \leq$ $t \leq 10$ in Figure 13 and Figure 14. Moreover, Figure 15 and Figure 16 demonstrate the same solutions with 2D plot for $0 \leq x \leq 50000$ at $t=5$. From Figure 15 and Figure 16, we can see that the wave frequency increases as $x$ approaches to zero.

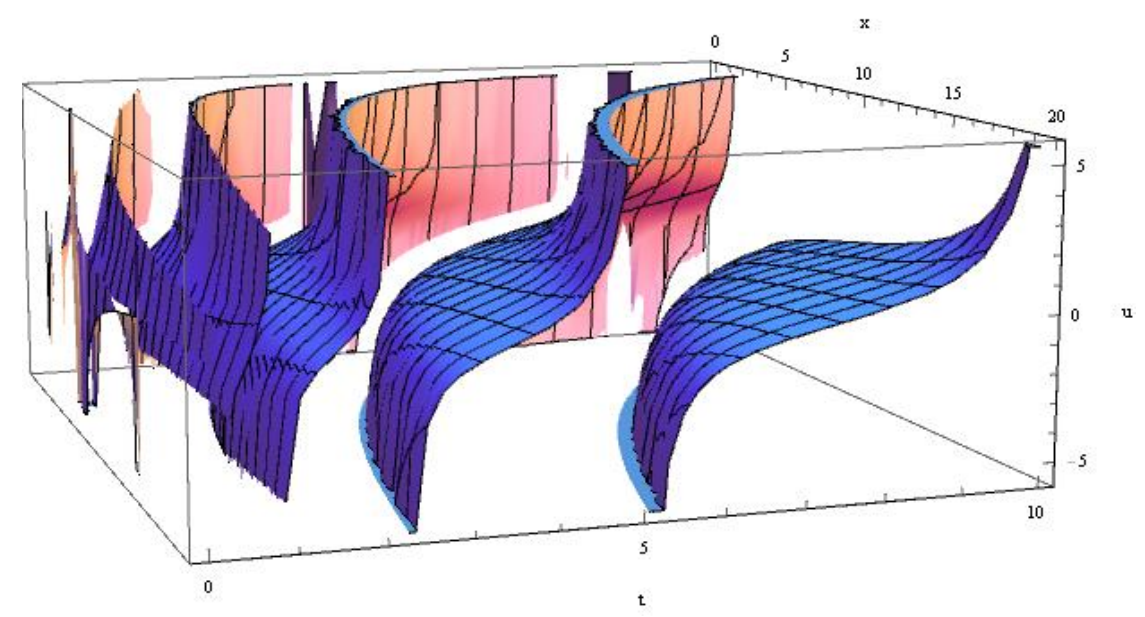

Figure 13. 3D plot of solution $u(x, t)=\tan (15 \sqrt[5]{t}+5 \sqrt[5]{x})$ 


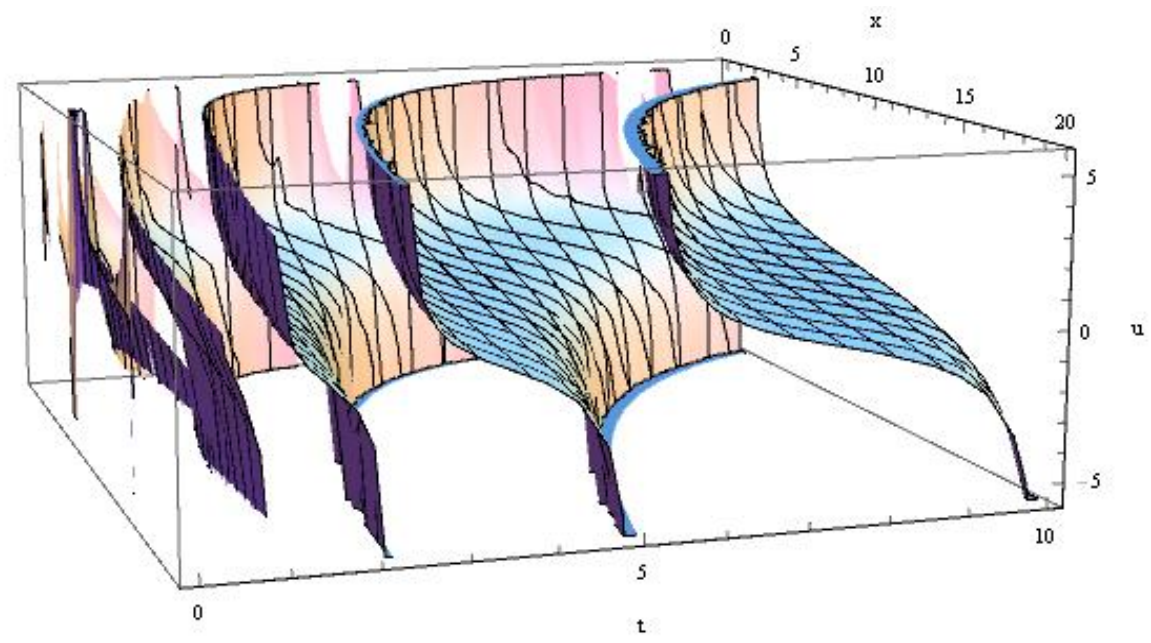

Figure 14. 3D plot of solution $u(x, t)=-\tan (15 \sqrt[5]{t}+5 \sqrt[5]{x})$

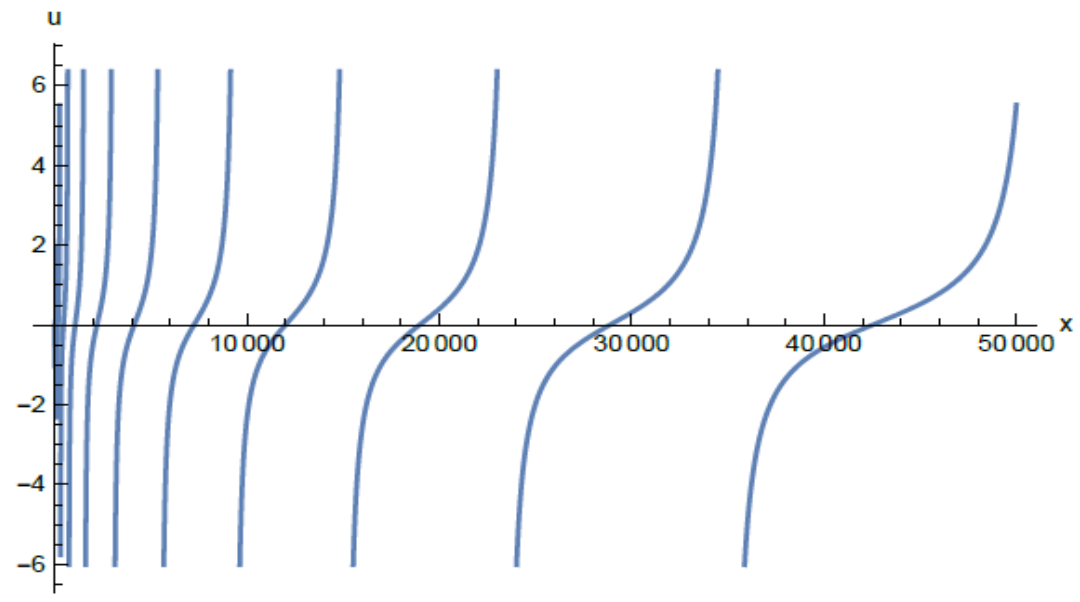

Figure 15. 2D plot of $u(x, t)=\tan (15 \sqrt[5]{5}+5 \sqrt[5]{x})$

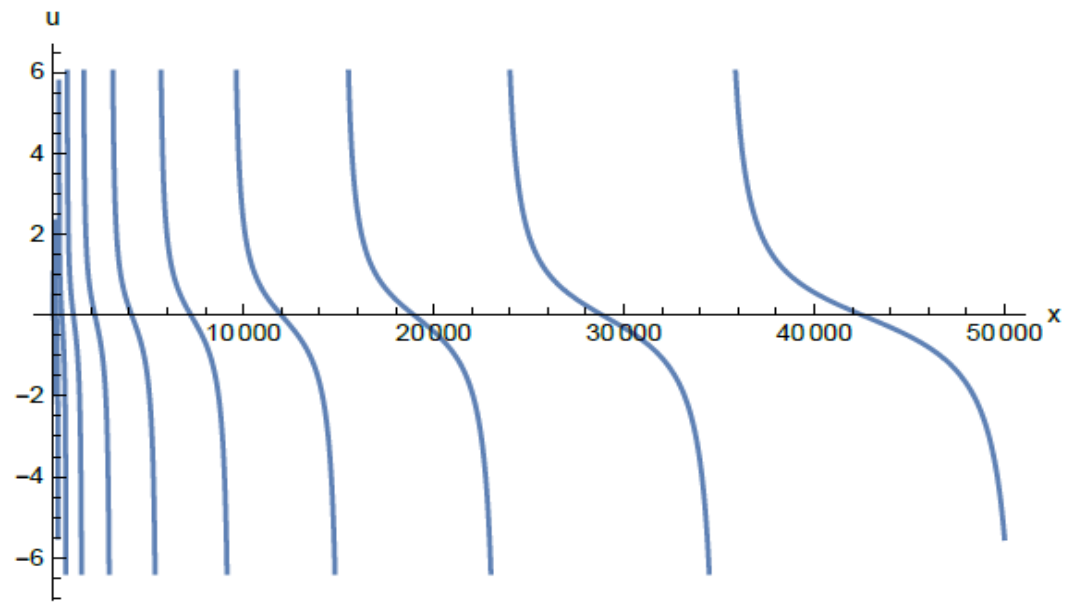

Figure 16. 2D plot of $u(x, t)=-\tan (15 \sqrt[5]{5}+5 \sqrt[5]{x})$ 


\section{CONCLUSIONS}

In this study, the exact solutions of the all of the time, space and space-time conformable fractional Phi-four equations, an analytic method has been developed using the Jacobi elliptic functions. This method is the first method in the literature and also this method is direct, quick and simple. The suggested method does not also need perturbation, linearization, boundary and initial conditions. Besides, by this method the solutions are found in a general form containing the hyperbolic, complex, rational and trigonometric functions, since the solutions include twelve Jacobi elliptic functions. Some of these solutions are solitary waves, such as kink like solutions illustrated in figures. Moreover, the solutions of the various methods such as sinecosine ansatz and tanh methods are covered by this method.

In the literature, the modified Kudryashov [19], exponential function [19], extended direct algebraic [20], mapping [21], modified mapping [21] and generalized Kudryashov [23] methods include the conformable derivatives with respect to time, while the $\left(G^{\prime} / G, 1 / G\right)$ expansion method [24] contains the conformable derivatives with respect to space and time. When compared with these methods, it is seen that more solutions are obtained by our method. Because 10 solutions containing rational, trigonometric and hyperbolic functions with the exponential function method, 4 solutions containing logarithmic function with the modified Kudryashov method, 33 solutions containing rational, trigonometric and hyperbolic functions with the extended direct algebraic method, 13 solutions containing trigonometric, hyperbolic functions with the mapping methods, 4 solutions containing rational function with the generalized Kudryashov method and 10 solutions aining rational, trigonometric and hyperbolic functions with the $\left(G^{\prime} / G, 1 / G\right)$-expansion method are obtained. However, there exist 192 type solutions for 30 different cases in our suggested method and also infinitely many solutions can be determined depending on the parameters $P, Q, R$ and $m$.

\section{REFERENCES}

[1] Lundquist, S., March, N. H. and Tosi, M. P., (1988), Order and Chaos in Nonlinear Physical Systems, Springer, New York.

[2] Calogero, F. and Degasperis, A., (1982), Spectral Transform and Solitons: Tools to Solve and Investigate Nonlinear Evolution Equations, New York: North-Holland.

[3] Wazwaz, A. M., (2007), Analytic study on nonlinear variants of the RLW and the PHI-four equations, Communications in Nonlinear Science and Numerical Simulation 12, 314-327.

[4] Wazwaz, A. M., (2005), Generalized forms of the phi-four equation with compactons, solitons and periodic solutions, Mathematics and Computers in Simulation, 69, 580-588.

[5] Alofi, A. S., (2013), Exact and Explicit Travelling Wave Solutions for the Nonlinear Partial Differential Equations, World Applied Sciences Journal, 21, 62-67.

[6] Soliman, A. A., (2007), Exact travelling wave solution of nonlinear variants of the RLW and the PHI-four equations, Physics Letters A, 368, 383-390.

[7] Deng, X., Zhao, M. and Li, X., (2009), Travelling wave solutions for a nonlinear variant of the PHI-four equation, Mathematical and Computer Modelling, 49, 617-622.

[8] Xiang, C., (2012), A note on exact travelling wave solutions for nonlinear PHI-four equation, Applied Mechanics and Materials, 4569-4572.

[9] Najafi, M., (2012), Using He's Variational Method to Seek the Travelling Wave Solution of PHI-Four Equation, International Journal of Applied Mathematical Research, 1 (4), 659-665. 
[10] Younis, M. and Zafar, A., (2013), The modified simple equation method for solving nonlinear Phi-Four equation, International Journal of Innovation and Applied Studies, 2, 661-664.

[11] Akter, J. and Akbar, M. A., (2015), Exact solutions to the Benney-Luke equation and the Phi-4 equations by using modified simple equation method, Results in Physics, 5, 125-130.

[12] Ehsani, F., Ehsani, F., Hadi, A. and Hadi, A., (2013), Analytical Solution of Phi-Four Equation, Technical Journal of Engineering and Applied Sciences, 3 (14), 1378-1388.

[13] Akbulut, A., Kaplan, M. and Tascan, F., (2016), Conservation laws and Exact Solutions of Phi-Four (Phi-4) Equation via the $\left(\mathrm{G}^{\wedge} / \mathrm{G}, 1 / \mathrm{G}\right)$ - Expansion Method, Naturforsch, 71 (5), 439-446.

[14] Zahra, W. K., (2017), Trigonometric B-Spline Collocation Method for Solving PHI-Four and Allen-Cahn Equations, Mediterranean Journal of Mathematics, 14, 122-141.

[15] Mahmud, F., Samsuzzoha, M. and Akbar, M. A., (2017), A generalized Kudrashov method to obtain exact travelling wave solutions of the PHI-four equation and the Fisher equation, Results in Physics, 7, 4296-4302.

[16] Islam, M. S., Khan, K. and Akbar, M. A., (2017), Application of the improved F-expansion method with Riccati equation to find the exact solution of the nonlinear evolution equations, Journal of the Egyptian Mathematical Society, 25, 13-18.

[17] Tariq, H. and Akram, G., (2017), New approach for exact solutions of time fractional Cahn-Allen equation and time fractional Phi-4 equation, Physica A, 473, 352-362.

[18] Alquran, M., Jaradat, H. M. and Syam, M. I., (2017), Analytical solution of the time-fractional Phi-4 equation by using modified residual power series method, Nonlinear Dyn, 90:2525-2529.

[19] Akram, G., Batool, F. and Riaz, A., (2018), Two reliable techniques for the analytical study of conformable time-fractional Phi-4 equation, Opt Quant Electron, 50:22.

[20] Rezazadeh, H., Tariq, H., Eslami, M., Mirzazadeh, M., and Zhou, Q., (2018), New exact solutions of nonlinear conformable time-fractional Phi-4 equation, Chinese Journal of Physics, 2, in press.

[21] Korpınar, Z., (2019), Some analytical solutions by mapping methods for non-linear conformable timefractıonal Phi-4 equation, Thermal Science, 23.

[22] Gao, W., Veeresha, P., Prakasha D. G., Baskonus, H. M. and Yel, G., (2020), New Numerical Results for the Time-Fractional Phi-Four Equation Using a Novel Analytical Approach, Symmetry, 12 (3), 478.

[23] Rahman, A., Alam, N. and Roshid, H., (2020), The generalized Kudryshov method implemented to the nonlinear conformable time-fractional PHI-Four equation, Annals of Pure and Appl. Mathematics 21 (1), 69-76.

[24] Sirisubtawee, S., Koonprasert, S., Sungnul, S. and Leekparn, T., (2019), Exact travelling wave solutions of the space-time fractional complex Ginzburg-Landau equation and the space-time fractional Phi-4 equation using reliable methods, Advances in Difference Equations, Doi: 10.1186/s13662-019-2154-9.

[25] Feng, Q., (2018), A new approach for seeking coefficient function solutions of conformable fractional partial differential equations based on the Jacobi elliptic equation, Chinese Journal of Physics, 56(6), 2817-2828.

[26] Tasbozan, O., Çenesiz, Y. and Kurt, A., (2016), New solutions for conformable fractional Boussinesq and combined KdV-mKdV equations using Jacobi elliptic function expansion method, The European Physical Journal Plus, 131(7), 244.

[27] Kurt, A., (2019), New periodic wave solutions of a time fractional integrable shallow water equation, Applied Ocean Research, 85, 128-135.

[28] El-Ganaini, S. and Al-Amr, M. O., (2019), New abundant wave solutions of the conformable space-time fractional (4+1)-dimensional Fokas equation in water waves, Computers \& Mathematics with Applications, 78(6), 2094-2106.

[29] Zayed, E. M. E. and Shohib, R. M. A., (2018), The unified sub-equation method and its applications to conformable space-time fractional fourth-order pochhammer-chree equation, Physics \& Astronomy International Journal, 2, 452-463. 
[30] Kumar, V. S., Rezazadeh, H., Eslami, M., Izadi, F. and Osman, M. S., (2019), Jacobi elliptic function expansion method for solving $\mathrm{KdV}$ equation with conformable derivative and dual-power law nonlinearity, International Journal of Applied and Computational Mathematics, 5(5), 127.

[31] Dascioglu, A., Culha, S., and Varol Bayram, D., (2017), New analytical solutions of the space fractional KdV equation in terms of Jacobi elliptic functions, New Trends in Mathematical Sciences, 5, No. 4, 232-241.

[32] Çulha, S. and Daşcıoğlu, A., (2019), Analytic solutions of the space-time conformable fractional KleinGordon equation in general form, Waves in Random and complex Media, 29, No. 4, 775-790.

[33] Çulha Ünal, S., Daşcıoğlu, A. and Varol Bayram, D., (2020), New exact solutions of space and time fractional modified Kawahara equation, Physica A: Statistical Mechanics and its Applications, 551, 124550.

[34] Ali, A. T., (2011), New generalized Jacobi elliptic function rational expansion method, Journal of Computational and Applied Mathematics, 235, 4117-4127.

[35] Khalil, R., Horani, M. A., Yousef, A. and Sababheh, M., (2014), A new definition of fractional derivative, Journal of Computational and Applied Mathematics, 264, 65-70.

[36] Abdeljawad, T., (2015), On conformable fractional calculus, Journal of Computational and Applied Mathematics, 279, 57-66.

[37] Erdelyi, A., Magnus, W., Oberhettinger, F. and Tricomi, F. G., (1993), Higher Transcendental Functions, vol. 2, McGraw-Hill: New York.

[38] Abramowitz, M. and Stegun, I. A., (1972), Handbook of Mathematical Functions with Formulas, Graphs, and Mathematical Tables, U.S. Government Printing Office: Washington, D.C.

[39] Remoissenet, M., (1993), Waves Called Solitons: Concepts and Experiments, Springer-Verlag, Berlin. 